Proceedings of the National Conference on Neutron Scattering and the Complementary Methods in the Investigations of the Condensed Phases, Chlewiska 2007

\title{
Energy Spectrum for the System of $N$ Ising Spins with Identical Spin-Spin Coupling $K / N$ - Anatomy of Phase Transition
}

\begin{abstract}
A. $\mathrm{CZACHOR}^{*}$
Institute of Atomic Energy, Świerk, 05-400 Otwock, Poland

Dedicated to Professor Jerzy Janik on the occasion of his 80th birthday

For the Kittel-Shore-Kac interspin coupling $K / N$ between $N$ Ising spins the ferromagnetic phase transition in specific heat vs. $T$ plot has appeared in literature as a purely mathematical phenomenon, via the exact calculation of the sum of states $Z(T)$ and subsequent differentiations with respect to temperature $T$. Physical nature of the transition remains in such derivation invisible. As it is expected to be related to the interaction/temperature competition in populating energy levels of the system, in this paper we construct the density of energy states $D(E)$ (or energy spectrum) of such systems, both for the ferromagnetic $(K>0)$ and antiferromagnetic $(K<0)$ coupling between spins. This allows one to see the essence of the difference between these systems as related to the discrete vs. quasi-continuous shape of the spectra at low energy states.
\end{abstract}

PACS numbers: $75.50 . \mathrm{Kj}, 65.40 .-\mathrm{b}$

\section{Introduction}

A system of $N$ spins $S=1 / 2$ coupled identically to each other is interesting because it can be and has been solved exactly in term of statistical physics [1-4]. Kac [4] has derived the partition function for the system of the Ising (or classical) spins coupled ferromagnetically (F) and using the saddle point method has found its free energy in the thermodynamic limit $N \rightarrow \infty$. It leads to the mean-field type of phase transition for magnetization of such system. Kittel and Shore [1] have shown that also for the system of vector spins $S=1 / 2$ there is such

*e-mail: a.czachor@cyf.gov.pl 
a phase transition, although it develops rather slowly for increasing $N$. (From now on we shall call systems of spins coupled identically - the KSK systems.) Al-Wahsh et al. [2] performed such a task for the KSK system of spins coupled antiferromagnetically $(\mathrm{AF})$. Global structure of the energy spectrum of the vector spin systems has been asserted by van der Sijs in group theory terms [3].

From the point of view of physical intuition the KSK model has serious deficiencies. It is dimension independent, while just dimensionality is essential for the critical indices of phase transitions. Besides, in order to reach the thermodynamic limit one has to take the interspin coupling to be of the form $K / N$, which is therefore the system-size-dependent coupling. These shortcomings of the model result in some unphysical predictions - e.g. the specific heat at $T \rightarrow 0$ does not go to zero. Nevertheless, being fully soluble, the model deserves attention - it allows one to test approximate methods of calculation for more realistic systems. Besides, the $K / N$ coupling is in a way the long-range interaction, which makes the system interesting even more.

To perform the calculations of the sum of states $Z$ one usually has to apply and rely on sophisticated mathematical methods. This complexity is somewhat obscuring the physical mechanism responsible for the temperature behavior of magnetic and thermal properties of such systems. One knows that it is the interplay between the coupling-driven ordering tendency and the disordering influence of temperature, which is responsible for the physical behavior of such systems. Therefore, it seems proper to find a method allowing one to set the anatomy of this competition in the appropriate scenery - in the space of energy levels and thus energy spectra $D(E)$ of such systems.

In this work we show that one can use the results of calculations given in [2] to derive for the Ising spins in a closed form the explicit form of the energy spectrum (density of states) for the KSK coupling between spins, at least at the absence of the external magnetic field. Some features of the specific heat vs. temperature plot for such a model magnet can be clearly explained in terms of the spectrum.

\section{Energy spectrum $D(E)$}

We shall follow the notation used in [2]. The energy levels for the system of $N$ Ising spins $S=1 / 2$ can be written as follows (for simplicity we shall deal here with even $N)$ :

$$
E_{L}=(I / 4)[L-(N-2 L)(N-2 L-1) / 2], \quad L=0,1 \ldots N,
$$

where the variable $L$ - the number of spins "inverted" with respect to the remaining $N-L$ spins - may be called a "quantum number" of this system. $I$ is the interspin coupling (called also the exchange integral).

We shall remember in the following that in the F-case $I>0$, while in the AF-case $I<0$. Figure 1 shows the dependence of $E$ on $L$. Extreme occurs at $L=N / 2$. Let us note that total number of different energy levels is $N+1$ and the energy range is $E_{N / 2}-E_{0}=I N^{2} / 8$. 


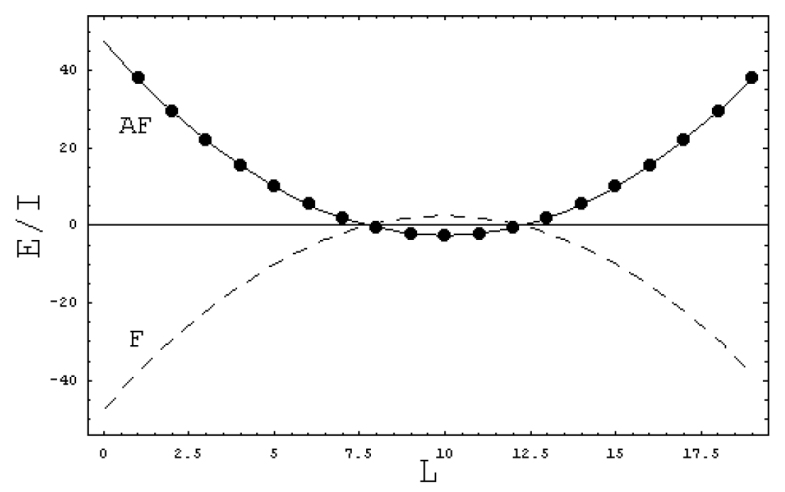

Fig. 1. Energy in the units $I$ vs. the "quantum number" $0<L<N$ of the system of $N$ spins coupled identically to each other with the "exchange integral" $I$, see Eq. (1). Antiferromagnetic coupling: $I<0$. Ferromagnetic coupling: $I>0$.

Equation (1) can be treated as the quadratic equation for the unknown variable $L$. We can easily solve it to represent the dependence of $L$ on the energy $E$ :

$$
L_{ \pm}=\left[N \pm(N-8 E / I)^{1 / 2}\right] / 2
$$

Degeneracy of the $L$-th level is just the number of ways in which one can turn $L$ spins up out of $N$ spins down, i.e. the Newton binomial coefficient

$$
d_{L}=B(N, L)=\frac{N !}{(N-L) ! L !} .
$$

It reflects the fact that the total number of different spin configurations for $N$ spins is $(1+1)^{N}$. Let us note that in the AF case for even $N$ the lowest energy level is at $L=N / 2$, its degeneracy being $N ! /[(N / 2) !]^{2}$. Having that, we can immediately write the formula for the density of states (energy spectrum) $D(E)$ :

$$
\begin{aligned}
D(E) & =\sum_{L} d_{L} \delta\left(E-E_{L}\right)=\sum_{L \neq N / 2} \frac{B(N, L)}{|L-N / 2|} \\
& \times\left\{\delta\left[L-L_{+}(E)\right]+\delta\left[L-L_{-}(E)\right]\right\}+d_{N / 2} \delta\left(E-E_{N / 2}\right),
\end{aligned}
$$

where the formula $\delta[x-f(y)]=\sum_{i} \delta\left[y_{i}-f^{-1}(x)\right] /\left|f^{\prime}\left(y_{i}\right)\right|, y_{i}$ being the roots of the equation $f(y)=x$, has been used. As we can see here the energy spectrum is essentially a series of $\delta$-function peaks at discrete values of the energy given by Eq. (1) - not a very deep insight into the problem yet.

\section{Profile function $P(E)$ for the KSK coupling $K / N$}

Now to make the system more tractable we introduce the KSK form of the coupling $[1,4]$ :

$$
I=K / N \text {, }
$$

where $K=K^{\mathrm{F}}>0$ means ferromagnetic coupling $(\mathrm{F})$, while $K=K^{\mathrm{AF}}<0$ is for the antiferromagnetic one $(\mathrm{AF})$. Let us note that now the total coupling of a given 
spin to all its neighbors is finite and goes to the energy value $K$ for $N$ growing indefinitely. One obtains instead of Eq. (2):

$$
L_{ \pm}(E)=[N \pm \sqrt{N(1-8 E / K)}] / 2 .
$$

These functions for both cases are shown in Fig. 2. Let us note that the total span of the energy range is now $N|K| / 8$.

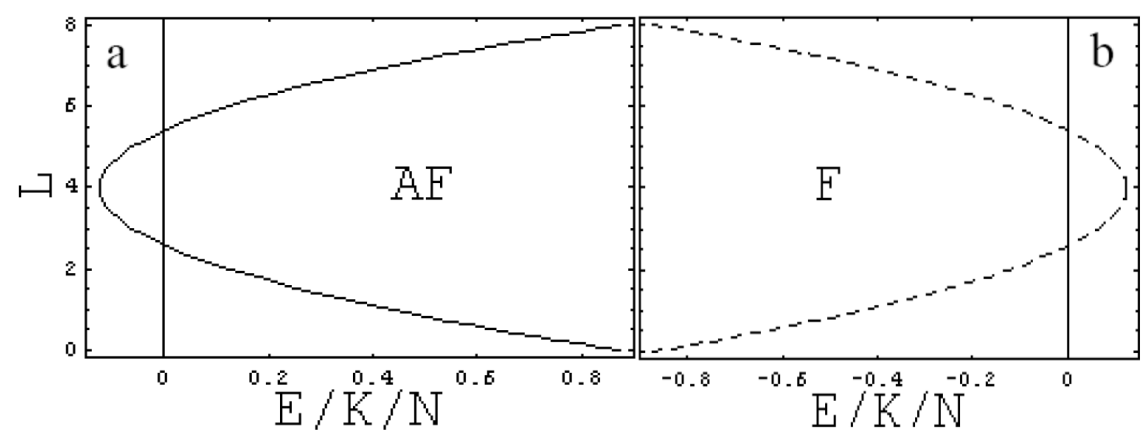

Fig. 2. The $L$ vs. $E$ plots for $N=20$ spins, Eq. (2), for the Kac interspin coupling $I=K / N$. Antiferromagnetic coupling $K^{\mathrm{AF}}<0$ (a), ferromagnetic: $K^{\mathrm{F}}>0(\mathrm{~b})$.

Let us now determine the limiting form of $d_{L}$ at large $N$. It is sufficient to use the well known Stirling approximation for the factorial

$$
\ln N !=(N+1 / 2) \ln N-N+\ln \sqrt{2 \pi} .
$$

Instead of $L$ we introduce into Eq. (7) the variable $m$ such that $L=N / 2-m$ and expand the result to second order in the "small" $m$. We receive an approximate formula for the Newton binomial coefficient $B(N, L)$ :

$$
\ln (B(N, N / 2-m))=\ln (B(N, N / 2))-2 m^{2}(1-1 / N) / N .
$$

It follows:

$$
B(N, L)=B(N, N / 2) \exp \left(-(8 \alpha / N)(N / 2-L)^{2}\right),
$$

where $\alpha=(1-1 / N) / 4$.

This approximate formula, although formally valid only for $m \ll N$, works already for $N=20$ amazingly well, at least to pictorial accuracy, in the whole range $0<L<N$, see Fig. 3. Only at the limits of this range the values of the Gaussian are too small, but it should play no role in possible integrations involving this function.

The last step is to insert into this formula the $L(E)$ function (6). After a simple manipulation one arrives for large $N$ at the continuous "profile" function $P(E)$ of the energy spectrum given by Eq. (4)

$$
\frac{B(N, L)}{|N / 2-L|} \cong C \frac{\exp (\alpha E / K)}{\sqrt{1-8 E / K}} \equiv P(E) .
$$

The energy spectrum can therefore be written as 


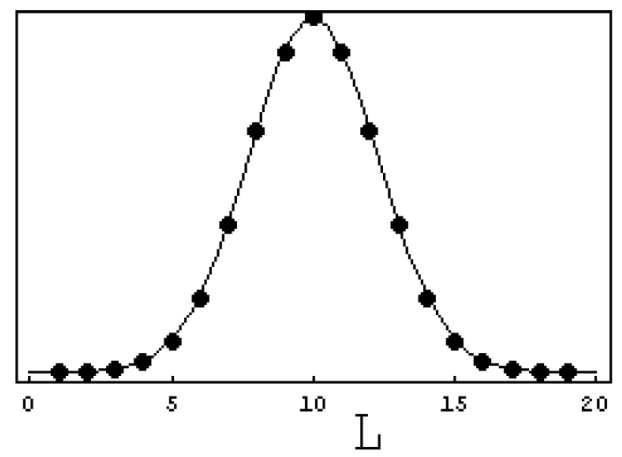

Fig. 3. Points - the Pascal-Newton binomial coefficients $B(N, L)$, Eq. (3); line - the corresponding Gauss curve, Eq. (9), for $N=20,0<L<N$.

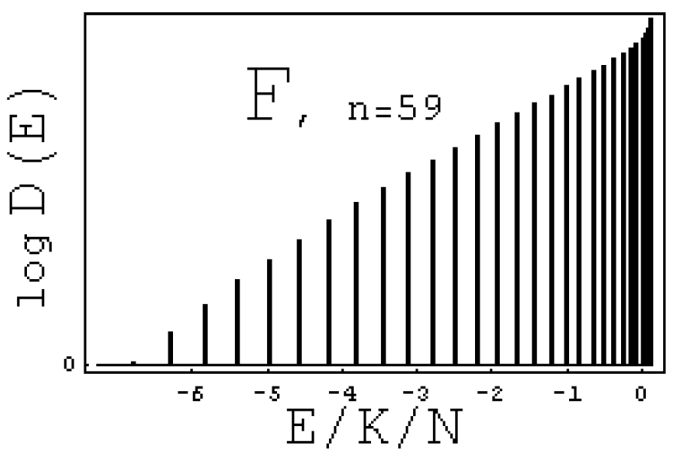

Fig. 4. Energy spectrum for the KSK ferromagnet, $N=59$. Logarithmic scale has been used to emphasize the rapid increase in the amplitude of subsequent $D(E)$ spikes for growing $E$, while the horizontal separation between the lowest levels is finite, of the order $K^{\mathrm{F}}$ for any $N$.

$$
D(E)=P(E) \sum_{L=0}^{N / 2}\left\{\delta\left[L-L_{+}(E)\right]+\delta\left[L-L_{-}(E)\right]\right\} .
$$

The constant $C$ in the $P(E)$ follows from the normalization condition $\int D(E) \mathrm{d} E=$ $2^{N}$; in the present context it is of secondary importance.

In this way we have arrived at the form of the energy spectrum we seek. The profile $P(E)$ can sometimes be treated as a continuous replica of the spectrum. Actually, it only provides the degeneracy weights to the $\delta$-functions in Eq. (11), representing the actual energy levels. Let us note that $P(E)$ near $E=K / 8$ shows the $x^{-1 / 2}$ singularity, which is integrable.

One should emphasize that the distribution of energy levels along the energy axis is highly non-uniform. For the F-case the distances between the energy levels close to the ground state are finite, of the order $K^{\mathrm{F}}$ for any $N$, while the degeneracy given by the $P(E)$ grows exponentially with increasing $E$, see Fig. 4 . This is why 
in order to evaluate the partition function for ferromagnets one must meticulously evaluate the relevant sums over discrete energy levels, as it has been done by Kac [4]; it is not allowed to substitute the sums involving $D(E)$ by integrations.

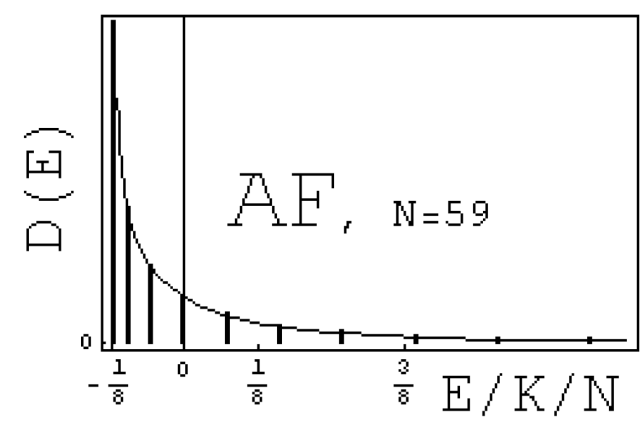

Fig. 5. Energy spectrum for the KSK antiferromagnet, $K^{\mathrm{AF}}<0$, in the vicinity and just above the ground state energy $E_{0}$, for $N=59$. Continuous line shows the profile function $P(E)$, Eq. (10), of the spectrum. Let us note that the (horizontal) separation between the energy levels becomes smaller — the density of levels declines - for $E \rightarrow E_{\min }$.

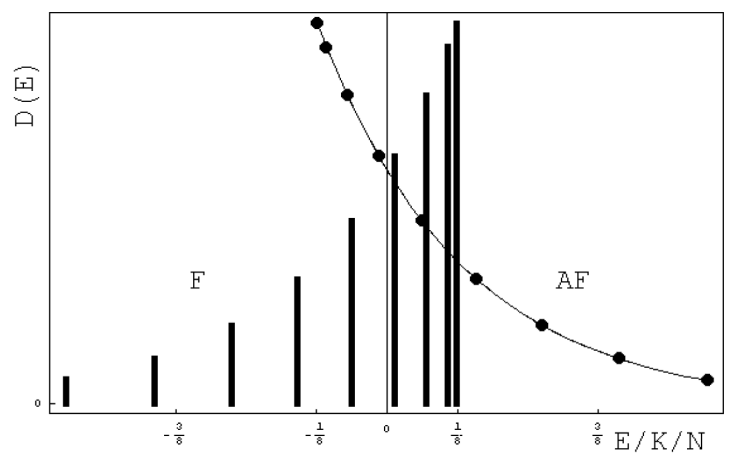

Fig. 6. Energy spectra, $N=59$, assuming $K^{\mathrm{F}}=-K^{\mathrm{AF}}=K$, which results in a mirror symmetry of both spectra. For evaluation of the sum of states the $F$-spectrum has to be treated as a sequence of discrete spikes, while the AF spectrum can in some cases be substituted by the continuous function $P(E)$, see right plot.

In the AF case for $N \rightarrow \infty$ the distances between lowest energy levels near the ground state tend to 0 , degeneracies of these levels approach each other, see Fig. 5, and one can sometimes substitute the $L$-sums by the $\mathrm{d} E$ integrations with the profile function $P(E)$. True, at the high energy the levels are again discrete, but as the relevant degeneracy weights also decline for growing $E$, the integration is often a right method. 
Both spectra $\mathrm{F}$ and $\mathrm{AF}$ are shown in Fig. 6 for some finite $N$ and in the energy ranges close to $E=K / 8$. As we can see, if the coupling is taken to be the same on the absolute value for both cases, they are symmetric with respect to each other. For $N \rightarrow \infty$ the lowest energy level in the F-case is $(1-N) K^{\mathrm{F}}$ and goes to minus infinity, while the highest one is $K^{\mathrm{F}} / 8$. In the case of the $\mathrm{AF}$ coupling the lowest energy level is $K^{\mathrm{AF}} / 8$ and the spectrum exponentially declines with $E \rightarrow \infty$.

\section{Thermodynamic functions}

Let us look at the thermodynamic consequences of the energy spectrum. In Eq. (11) the function $P(E)$ in front of the sum provides a continuous envelope for the spectrum, but the distribution of relevant energy points along the energy axis is both discrete and non-uniform.

The last fact is of primary importance, at least for the ferromagnetic spin ordering. Let us consider it in some detail. The distance between lowest energy levels is here finite for any $N$, of the order $K^{\mathrm{F}}$. Such a finite separation between the ground state energy and higher energy levels in the energy spectrum is in fact essential for many physical systems, because it usually secures the accord with the Nernst theorem (see e.g. [5]) for the specific heat $c_{V}(T)$ :

$$
C_{V}(T) \underset{T \rightarrow 0}{\longrightarrow} 0 .
$$

However, on inspecting the Kac results [4] following his evaluation of the partition function one finds out that the Ising KSK ferromagnet is in this temperature limit unphysical - the specific heat tends in this limit to a constant value. Similar problems arise within this model for the magnetic properties at low temperatures. When looking at the energy spectrum we can see no reason for such defects. The Kac method of evaluating the partition function and free energy seems to be exact, but it seems that there may be some convergence problems at the low temperature limit.

The Weiss theory of ferromagnetism, although in principle derivable for the same model of spins, predicts at low temperatures a reasonable behavior. Initially postulated by Weiss as an ingenious guess, it can also be derived using the Gibbs procedure. In this free energy approximation one extracts from the sum of states $Z(T)$ (by calculating the extremum with respect to magnetization) the subsum which is "overwhelmingly large" [6]. Alternatively, one can speak of the Bragg-Williams treatment of the Ising spins, where the system is considered as a "chemical" mixture of spins up an down [7]. Paradoxically, the approximation is in this case more "physical" than the exact theory. The density of states involved in such "limited" sum of states is not known; presumably it shows essentially lower degeneracy of the lowest energy states than the exact $D(E)$ shown here.

At elevated temperatures the KSK model rigorously leads to the ferromagnetic phase transition and we can interpret it in terms of the density of states $D(E)$. The dramatic growth of the degeneracy level vs. $E$ in the evaluation of the 
sum of states $Z(T)$ is for increasing temperature $T$ balanced more and more by the exponentially declining Gibbs factor $\exp \left(-E / k_{\mathrm{B}} T\right)$. At sufficiently high temperatures the system occupies mainly the group of energy levels in the infinitesimal energy range close to the highest energy level $K / 8$, where the $P(E)$ function shows a spike, see Fig. 4. It leads to the ferromagnetic phase transition at $T_{\mathrm{C}}-$ a spike in the specific heat - which is important merit of the Kac approach. At temperature above the $T_{\mathrm{C}}$ the internal energy of such Ising ferromagnet is practically constant and equal to $K / 8$, so its first derivative with respect to temperature $c_{V}=0$.

For the KSK antiferromagnet the situation is mathematically different but physically similar. The distances between the lowest energy levels are of the order $K^{\mathrm{AF}} / N$, thus the spectrum for $N \rightarrow \infty$ becomes quasi-continuous. It is therefore reasonable to evaluate the thermodynamic functions for this case using the continuous form of the energy spectrum (10), see continuous line in Fig. 4, and turning the relevant sums into integrals. The sum of states is

$$
\begin{aligned}
Z_{N} & =\sum_{L} \frac{B(N, L)}{|N / 2-L|} \exp \left(-\beta E_{L}\right) \\
& \cong C \int_{-K^{\mathrm{AF}}}^{(N-1) K^{\mathrm{AF}}} \frac{\exp (\alpha E / K)}{\sqrt{1-8 E / K}} \exp (-\beta E) \mathrm{d} E \\
& \approx \frac{K}{8} \exp \left(\frac{\alpha+\beta K}{8}\right) \int_{0}^{N} \frac{\exp \left(-\frac{\alpha+\beta K}{8} x\right)}{\sqrt{x}} \mathrm{~d} x \\
& \underset{N \rightarrow \infty}{\longrightarrow} \frac{\sqrt{2 \pi} K}{4} \frac{\exp \left(\frac{\alpha+\beta K}{8}\right)}{\sqrt{\alpha+\beta K}},
\end{aligned}
$$

where $\beta=k_{\mathrm{B}} T$ and here $K \equiv K^{\mathrm{AF}}$. The energy is $E_{N}=-\partial Z_{N} / \partial \beta / Z_{N}$. The specific heat per one spin $c_{V}=(1 / N) \partial E_{N} / \partial T$ is

$$
C_{V} \approx \frac{1}{2 N} \frac{K}{(\alpha T+K)^{2}} \underset{N \rightarrow \infty}{\longrightarrow} 0 .
$$

Thus $c_{V}(T)=0$ for the KSK antiferromagnet at all temperatures, as it has been derived exactly within the independent theory [2]. Indirectly this agreement proves that the continuous profile function $P(E)$ can be treated as the density of states $D^{\mathrm{AF}}(E)$ for the KSK antiferromagnet. Physically, it is related to the fact that in the AF case for $N \rightarrow \infty$ the chance of promotion of the system to a higher energy effectively declines to zero, because the ratio of degeneracy of a higher energy level to the degeneracy of a lower one becomes in this limit infinitesimally small, see also discussion in Ref. [2].

\section{Conclusions}

1. The density of states $D^{\mathrm{AF}}(E)$ for the KSK antiferromagnet at $N \rightarrow \infty$ is given by the quasi-continuous profile function $P(E)$. It shows in the energy range $\left(K^{\mathrm{AF}} / 8, \infty\right)$ practically an exponential decline vs. $E$. The summation 
over $L$ in the sum of states $Z$ and other thermodynamic functions can be in this $(\mathrm{AF})$ case substituted by the $\mathrm{d} E$ integration.

2. The discrete nature of the density of states $D^{\mathrm{F}}(E)$ is essential for the right calculation of the thermodynamic functions for ferromagnets at elevated and high temperatures - one has to evaluate the relevant sums $\sum_{L} \ldots$ involving the $\delta\left[L-L_{ \pm}(E)\right]$ spikes, not the above mentioned integral.

3. The KSK model in case of ferromagnetic coupling of the Ising spins becomes at $T \rightarrow 0$ physically doubtful, while the mean-field model of Weiss, being formally an approximation within the KSK model, displays a physically acceptable behavior in this limit.

\section{References}

[1] C. Kittel, H. Shore, Phys. Rev. A 138, 1165 (1965).

[2] H. Al-Wahsh, M. Urban, A. Czachor, J. Magn. Magn. Mater. 185, 144 (1998).

[3] A.J. van der Sijs, Phys. Rev. B 48, 7125 (1993).

[4] M. Kac, in: Statistical Physics, Phase Transitions and Superfluidity, Eds. M. Chretien, E.P. Gross, S. Desser, Vol. 1, Gordon and Breach, New York 1968, p. 241.

[5] L. Landau, E. Lifshitz, Statistical Physics, PWN, Warszawa 1959 (in Polish).

[6] R.H. Brout, in Ref. [4], p. 3.

[7] G.H. Wannier, Elements of Solid State Physics, The University Press, Cambridge 1959. 